\title{
Delivery of Resveratrol, a Red Wine Polyphenol, from Solutions and Hydrogels via the Skin
}

\author{
Chi-Feng Hung, ${ }^{a}$ Yin-Ku Lin, ${ }^{b, c}$ Zih-Rou HuAnG, ${ }^{d}$ and Jia-You FAnG ${ }^{*, d}$ \\ ${ }^{a}$ School of Medicine, Fu Jen Catholic University; Taipei County 242, Taiwan: ${ }^{b}$ Graduate Institute of Clinical Medical \\ Sciences, Chang Gung University; ${ }^{c}$ Department of Traditional Chinese Medicine, Center for Traditional Chinese \\ Medicine, Chang Gung Memorial Hospital; and ${ }^{d}$ Pharmaceutics Laboratory, Graduate Institute of Natural Products, \\ Chang Gung University; Kweishan, Taoyuan 333, Taiwan. \\ Received October 29, 2007; accepted February 4, 2008; published online February 5, 2008
}

\begin{abstract}
Resveratrol, the main active polyphenol in red wine, has been demonstrated to show benefits against skin disorders. The bioavailability of orally administered resveratrol is insufficient to permit high enough drug concentrations for systemic therapy. In this study, we examined the feasibility of the topical/transdermal delivery of resveratrol. The effects of vehicles on the in vitro permeation and skin deposition from saturated solutions such as aqueous buffers and soybean oil were investigated. The general trend for the delivery from solutions was: $\mathrm{pH} 6$ buffer $=$ pH 8 buffer $>10 \%$ glycerol formal in pH 6 buffer $>$ pH 9.9 buffer $>$ pH 10.8 buffer $>$ soybean oil. A linear relationship was established between the permeability coefficient $\left(K_{\mathrm{p}}\right)$ and drug accumulation in the skin reservoir. Viable epidermis/dermis served as the predominant barrier for non-ionic resveratrol permeation. On the other hand, both the stratum corneum (SC) and viable skin acted as barriers to anionic resveratrol. Several prototype hydrogel systems were also studied as resveratrol vehicles. The viscosity but not the polarity of the hydrogels controlled resveratrol permeation/deposition. Piceatannol, a derivative of resveratrol with high pharmacological activity, showed 11.6-fold lower skin permeation compared to resveratrol. The safety profiles of resveratrol suggested that the hydrogel caused no SC disruption or skin erythema. It was concluded that delivery via a skin route may be a potent way to achieve the therapeutic effects of resveratrol. This is the first report to establish the permeation profiles for topically applied resveratrol.
\end{abstract}

Key words resveratrol; skin; topical delivery; transdermal delivery; hydrogel

Resveratrol is a naturally occurring polyphenolic compound which has received much attention in the prevention of and therapy for cardiovascular diseases and cancers (Fig. 1). ${ }^{1)}$ Red grapes and wines contain considerable amounts of resveratrol. Resveratrol has many benefits for the skin. Topically applied resveratrol has been shown to possess strong antiproliferative and chemopreventive properties against skin carcinogenesis. $^{2-4)}$ Resveratrol affords substantial protection against skin damage from ultraviolet B (UVB) exposure. ${ }^{5,6)}$ Resveratrol shows antimicrobial activity against dermatophytes and herpes simplex virus. ${ }^{7,8)}$ Resveratrol can also be used in the dermocosmetic field because of its role as a phytoestrogen based on its ability to activate estrogen receptors. ${ }^{9)}$

The oral bioavailability of resveratrol is poor, leading to an irrelevant in vivo effect with oral administration compared to its powerful in vitro efficacy. ${ }^{10)}$ Resveratrol also has a short initial half-life ( $8-14 \mathrm{~min})$ and is extensively metabolized in the body. ${ }^{11)}$ Several phase I clinical trials are currently underway for oral resveratrol in humans at doses of as high as $7.5 \mathrm{~g}$ per day. This would require $2.7 \mathrm{~kg}$ of resveratrol a year at a current cost of $c a$. US\$6800. Therefore it is becoming increasingly important to find ways to improve the bioavailability of and block the metabolism of resveratrol. Delivery of resveratrol via the skin is an attractive alternative to oral dosing for both topical and transdermal applications.

No evidence currently exists in the literature to support the notion that a sufficient amount of resveratrol can be delivered to produce therapeutic benefits via a skin route. Designs of optimal formulations for resveratrol permeation via the skin are also lacking. The purposes of this study were to establish basic profiles of resveratrol permeability into and across the skin. The optimal $\mathrm{pH}$ and co-solvent of the vehicles for resveratrol were investigated for better permeation. The present study utilized Franz cells to explore the in vitro skin permeation of resveratrol. The drug amount retained within the skin reservoir was determined by in vitro and in vivo methods. Various skin membranes, including nude mouse skin, stratum corneum (SC)-stripped skin, delipidized skin, and cellulose membranes, were used as permeation barriers to elucidate the mechanisms and pathways. One particular problem common to drug delivery systems designed for use on the skin is the poor retention at the site of application. This problem can be resolved by incorporating bioadhesive hydrogels within the system. Hence, the ability of a series of hydrogel formulations to deliver topically applied resveratrol was examined. The permeability of piceatannol (Fig. 1), a resveratrol analogue with considerably higher activity, was also evaluated in this study.

\section{MATERIALS AND METHODS}

Materials Resveratrol, piceatannol, $\alpha$-terpineol, oleic acid, glycerol formal, and Nile red were purchased from Sigma Chemical (St. Louis, MO, U.S.A.). Carbopol ${ }^{\circledR} 940$ and

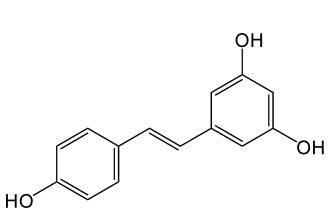

Resveratrol

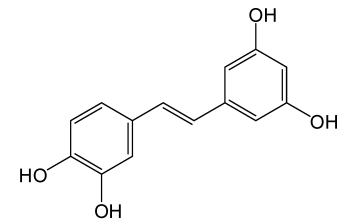

Piceatannol
Fig. 1. Chemical Structures of Resveratrol and Piceatannol 
Ultrez20 were supplied by B.F. Goodrich (Cleveland, $\mathrm{OH}$, U.S.A.). The ammonium acryloyldimethyltaurate/vinylporrolidone copolymer (AVC) was obtained from Clariant International (Frankfurt, Germany). Hydroxyethyl cellulose (HEC), hydroxypropyl cellulose (HPC, 1000 - $4000 \mathrm{cp}$ ), and carboxylmethyl cellulose sodium $(\mathrm{CMCNa})$ were purchased from Wako Chemical (Tokyo, Japan). Hydroxypropyl methylcellulose (HPMC, Metolose ${ }^{\circledR}$ 60SH-4000) was from Shin-Etsu Chemical (Tokyo, Japan). The cellulose membrane (with a molecular weight cutoff of 6000-8000) was obtained from Membrane Filtration Products (Seguin, TX, U.S.A.).

Determination of Resveratrol Solubility in Vehicles An excess amount of resveratrol was added to $1 \mathrm{ml}$ of selected buffers or oil, and shaken reciprocally at $37^{\circ} \mathrm{C}$ for $24 \mathrm{~h}$. The suspension was centrifuged at $10000 \mathrm{rpm}$ for $10 \mathrm{~min}$, and the drug concentration in the supernatant was determined using high-performance liquid chromatography (HPLC) after the appropriate dilution.

HPLC Analytical Method The HPLC system for both resveratrol and piceatannol included a Hitachi (Tokyo, Japan) L-7110 pump, a Hitachi L-7200 sample processor, and a Hitachi L-7400 UV/visible detector. A 25-cm-long, 4-mm inner diameter stainless RP-18 column (Merck, Darmstadt, Germany) was used. The mobile phase was a methanol:pH 2.6 aqueous solution adjusted with acetic acid $(45: 55)$ at a flow rate of $1.0 \mathrm{ml} / \mathrm{min}$. The $\mathrm{UV} /$ visible detector was set at $310 \mathrm{~nm}$.

Preparation of Hydrogels Calculated amounts of polymer and resveratrol were added into a half volume of $\mathrm{pH} 6$ buffer with continuous stirring for $1 \mathrm{~h}$. The other half-volume of $\mathrm{pH} 6$ buffer was added after $24 \mathrm{~h}$ incubation at room temperature. The hydrogel formulations were used to perform the in vitro or in vivo experiments after $24 \mathrm{~h}$ of preparation.

Determination of Hydrogel Viscosity The viscosity of the hydrogels was measured using a programmable viscometer (model DV-II+, Brookfield, U.S.A.). Readings were recorded $30 \mathrm{~s}$ after the measurement was made, when the level had stabilized.

Determination of Hydrogel Polarity The hydrophobic fluorescent marker, Nile red $\left(2.5 \times 10^{-5} \%\right.$, w/w), was used as the model solute, and the molecular environment (polarity) was elucidated by fluorometric spectroscopy due to the solvatochromism of Nile red. Emission fluorescence spectra were determined with an F-2500 fluorescence spectrophotometer (Hitachi). The spectra of the aqueous solutions of polymers loaded with Nile red were recorded at room temperature with both slit widths set to $10 \mathrm{~nm}$. The excitation wavelength was fixed at $546 \mathrm{~nm}$, and emission spectra were recorded from 550 to $700 \mathrm{~nm}$ at a scanning speed of $300 \mathrm{~nm} / \mathrm{min}$

Preparation of Skin Membranes Female nude mice ( $c a .8$ weeks old) were sacrificed, and full-thickness skin was excised from the dorsal region. To obtain SC-stripped skin, adhesive tape $\left(\mathrm{Scotch}^{\circledR}, 3 \mathrm{M}\right.$, U.S.A.) was applied to the skin with uniform pressure and then removed. This procedure was repeated 20 times. Delipidized skin was prepared by pretreating the SC with chloroform-methanol $(2: 1, \mathrm{v} / \mathrm{v})$ for $1 \mathrm{~h}$. Five percent $\alpha$-terpineol or oleic acid in a $25 \%$ ethanol/water vehicle was used to pretreat skin mounted on Franz cells for $2 \mathrm{~h}$, followed by the in vitro permeation experiment.
In Vitro Degradation Test The in vitro sensitivity to enzymatic degradation of resveratrol and piceatannol was carried out using skin homogenate. The nude mice skin (150 mg) was homogenized by $\mathrm{pH} 7.4$ citrate-phosphate buffer $(850 \mu \mathrm{l})$, then centrifuged for $10 \mathrm{~min}$ at $9300 \mathrm{rpm}$. The supernatant was withdrawn as the skin homogenate. Each drug was dissolved in $\mathrm{pH} 6$ buffer to give a saturated concentration. A solution of skin homogenate with a volume of $300 \mu \mathrm{l}$ was added to $200 \mu \mathrm{l}$ of the drug solution. The mixture was incubated at $37^{\circ} \mathrm{C}$. At predetermined intervals, the reaction mixture was withdrawn and immediately determined by HPLC.

In Vitro Skin Permeation The cellulose membrane and skins with or without treatments were mounted on the receptor compartment of a Franz cell with the SC side facing upwards into the donor compartment. Five and a half milliliters of $3: 7(\mathrm{v} / \mathrm{v})$ ethanol-pH 7.4 buffer was used as the receptor medium. The donor compartment was filled with $0.5 \mathrm{ml}$ vehicle containing resveratrol or piceatannol at a dose of saturated solubility. The available diffusion area between compartments was $0.785 \mathrm{~cm}^{2}$. The stirring rate and temperature were kept at $600 \mathrm{rpm}$ and $37^{\circ} \mathrm{C}$, respectively. At appropriate intervals, $300-\mu 1$ aliquots of the receptor medium were withdrawn and immediately replaced with an equal volume of fresh buffer.

At the end of the in vitro experiment, the skin was removed from the cell and washed briefly in water and methanol. The skin was then weighed, cut with scissors, positioned in a glass homogenizer containing $1 \mathrm{ml}$ of methanol, and homogenized for $5 \mathrm{~min}$. The resulting solution was centrifuged for $10 \mathrm{~min}$ at $10000 \mathrm{rpm}$ and then filtered through a PVDF membrane with a pore size of $0.45 \mu \mathrm{m}$. All samples were analyzed by HPLC.

In Vivo Skin Permeation For the in vivo experiment, $c a$. 8-week-old nude mouse was used. A glass cylinder with an available area of $0.785 \mathrm{~cm}^{2}$ was placed on the dorsal skin with glue (Instant Super Glue ${ }^{\circledR}$, Kokuyo, Japan). An aliquot of $0.2 \mathrm{ml}$ of vehicle with resveratrol was added to the cylinder. The application time of the vehicle was $12 \mathrm{~h}$. The application region of the skin was excised at the end of the experiment. The procedure for extraction of the drug from the skin was the same as for the in vitro experiment.

In Vivo Transepidermal Water Loss (TEWL) and Skin Erythema A 0.6- $\mathrm{ml}$ aliquot of vehicle with resveratrol was spread uniformly over a sheet of non-woven polyethylene cloth $(1.5 \times 1.5 \mathrm{~cm})$, which was then applied to the back area of a nude mouse. The polyethylene cloth was fixed with Tegaderm ${ }^{\circledR}$ adhesive dressing (3M) and Fixomull ${ }^{\circledR}$ stretch adhesive tape (Beiersdorf AG, Germany). After $24 \mathrm{~h}$, the cloth was removed, and the treated skin area was swabbed clean with a cotton wool swab. After withdrawal of the vehicle for $30 \mathrm{~min}$, TEWL and colorimetric parameters were measured. TEWL was recorded using a Tewameter ${ }^{\circledR}$ (TM300, Courage \& Khazaka, Germany). The TEWL was automatically calculated and expressed in $\mathrm{g} / \mathrm{m}^{2} / \mathrm{h}$. A spectrocolorimeter (CD100, Yokogawa Electrical, Japan) was used to measure the skin erythema $\left(a^{*}\right)$. The instrument recorded color reflectance three-dimensionally $\left(\mathrm{L}^{*}, \mathrm{a}^{*}, \mathrm{~b}^{*}\right)$ as recommended by CIE (Commission Internationale de l'Eclairage). When recording the color values, the measuring head was held perpendicular to the dorsal skin of the mouse and the aperture was fitted 
with an applicator, to avoid compression of subcutaneous capillaries. After pressing the measuring button, the obtained reading appeared within a few seconds on the display. An adjacent untreated site was used as a baseline standard for each determination. The temperature and relative humidity in the laboratory were kept at $26^{\circ} \mathrm{C}$ and $55 \%$, respectively.

Data Analysis To calculate the permeation parameters of Fick's law from the plot of the permeated amount vs. time, a graph was plotted as shown in Fig. 1. The flux value at the steady-state was determined and expressed per unit of diffusion area in $\mathrm{nmol} / \mathrm{cm}^{2} / \mathrm{h}$ by a linear regression calculation from the slope of the linear portion of the cumulative amount-time profiles. The permeability coefficient $\left(K_{\mathrm{p}}, \mathrm{cm} / \mathrm{h}\right)$ was calculated from the flux divided by the applied drug concentration in the donor compartment. The effect of each treatment, such as SC-stripping, delipidization, and enhancers, was represented by Ratio $_{\mathrm{PC}}$ which was calculated as: Ratio $_{\mathrm{PC}}=K_{\mathrm{p}}$ with treatment $/ K_{\mathrm{p}}$ without treatment (control).

A statistical analysis of differences among the various treatments was performed using unpaired Student's $t$-test. A 0.05 level of probability was taken as the level of significance. An analysis of variance (ANOVA) test was also used if necessary.

\section{RESULTS}

In Vitro Skin Permeation of Resveratrol from Solution The $\mathrm{pH}$ of the vehicle has been shown to be one of the major variables that can influence the diffusivity of drugs. ${ }^{12}$ ) Resveratrol's structure has multiple protonation sites. The acidic-dissociation constants were determined to be $\mathrm{p} K_{\mathrm{a} 1}=$ $8.01, \mathrm{p} K_{\mathrm{a} 2}=9.86$, and $\mathrm{p} K_{\mathrm{a} 3}=10.50 .{ }^{13)}$ In this work, the effect of $\mathrm{pH}$ of buffers on skin permeation of resveratrol was respectively examined from $\mathrm{pH} 6$ to 10.8 . The ionization of resveratrol increased following an increase in the $\mathrm{pH}$. Drug concentrations of saturated solutions in buffer were determined as detailed in Table 1 . The solubility of resveratrol was low at $\mathrm{pH}$ values below $\mathrm{p} K_{\mathrm{a} 1}$, and it increased from $\mathrm{pH} 8$ to 10.8 , which were above $\mathrm{p} K_{\mathrm{a} 1}$.

In vitro permeation of resveratrol through excised skin was investigated for all buffers ranging from $\mathrm{pH} 6$ to 10.8 . Resveratrol could maintain its prototype after skin permeation based on the HPLC chromatograms, thus assuring the biological activity. Moreover, no resveratrol degradation was observed in skin homogenate during a 12-h duration (in vitro degradation test). The flux and $K_{\mathrm{p}}$ values are given in Table 1 , and a representative example is shown in Fig. 2. Linear regression analysis of the permeation data was done to deter- mine the proper order of release. Zero, first, and Higuchi model equations were applied to all in vitro permeation results. The results show that the permeation of resveratrol was almost linear when using the zero reder equation ( $r>0.97$ for all curves). The $\mathrm{pH}$ of the vehicle had a significant effect on the transport of resveratrol $(p<0.05)$. The resveratrol flux increased in the order of $\mathrm{pH} 8>\mathrm{pH} 6>\mathrm{pH} 9.9>\mathrm{pH} 10.8$. On the other hand, the $K_{\mathrm{p}}$ of the drug was greatest from the saturated solution at $\mathrm{pH} 6$ and least from that at $\mathrm{pH} 10.8$ $(p<0.05)$. The other barrier examined for resveratrol transport was a synthetic cellulose membrane. As shown in Table 1 , it was apparent that the diffusion through the cellulose membrane was significantly higher $(p<0.05)$ than that through skin. The amounts of drug released by the various $\mathrm{pH}$ values were comparable $(p>0.05)$, except that $\mathrm{pH} 10.8$ showed lower drug liberation.

For topical formulations, the drug skin content is considered an important parameter, and in the present study, the skin deposition was determined at the end of the in vitro experiment. The drug was extracted from the skin to examine the drug accumulation as shown in Table 1. The deposition of resveratrol was noted to have sharply decreased as the $\mathrm{pH}$ of the donor compartment increased.

Previous studies suggested that glycerol formal exhibits a great solubility capacity for resveratrol. ${ }^{10,14)}$ A $10 \%$ glycerol formal/pH 6 buffer was used as the vehicle to examine resveratrol permeation. As shown in Table 1, this vehicle showed the highest flux among all formulations tested $(p<0.05)$. However, the $K_{\mathrm{p}}$ from this system was lower than that from the $\mathrm{pH} 6$ and 8 buffers because of the great solubil-

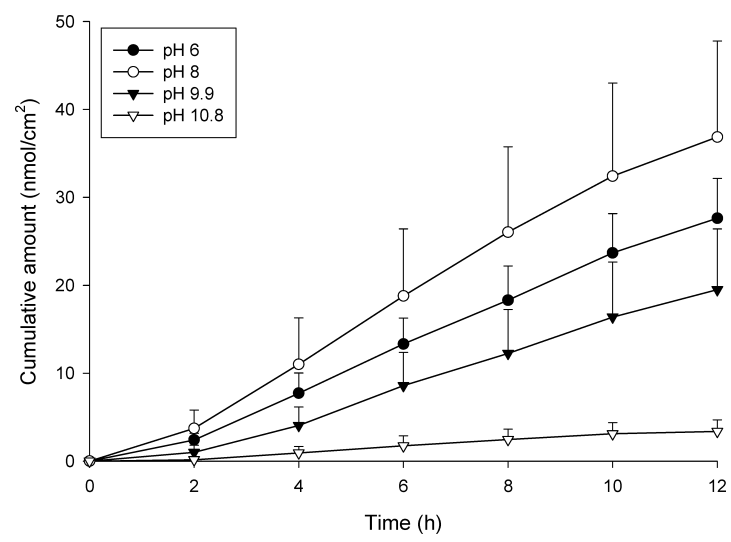

Fig. 2. Cumulative Amount versus Time Profiles of in Vitro Topical Resveratrol Application Permeating across Nude Mouse Skin from Aqueous Buffers with Different $\mathrm{pH}$ Values

All data represent the means of four experiments \pm S.D.

Table 1. Solubility, Permeation Data, and Skin Deposition of Resveratrol from Aqueous Solutions with Various pH Values and Oils

\begin{tabular}{|c|c|c|c|c|c|}
\hline Vehicle & $\begin{array}{l}\text { Solubility } \\
(\mathrm{nmol} / \mathrm{l})\end{array}$ & $\begin{array}{c}\text { Flux } \\
\left(\mathrm{nmol} / \mathrm{cm}^{2} / \mathrm{h}\right)\end{array}$ & $\begin{array}{l}\text { Permeability coefficient } \\
\left.\qquad\left(\mathrm{cm} / \mathrm{h} \times 10^{-4}\right)^{a}\right)\end{array}$ & $\begin{array}{l}\text { Release rate } \\
\left(\mathrm{cm} / \mathrm{h} \times 10^{-4}\right)^{b)}\end{array}$ & $\begin{array}{l}\text { Skin deposition } \\
(\mu \mathrm{g} / \mathrm{g})^{c)}\end{array}$ \\
\hline pH 6 buffer & $0.22 \pm 0.05$ & $1.59 \pm 0.08$ & $72.16 \pm 3.54$ & $464.93 \pm 41.79$ & $303.03 \pm 41.35$ \\
\hline pH 8 buffer & $0.23 \pm 0.002$ & $1.86 \pm 0.21$ & $79.73 \pm 9.03$ & $441.26 \pm 35.67$ & $273.62 \pm 52.38$ \\
\hline pH 9.9 buffer & $0.60 \pm 0.003 * *$ & $0.90 \pm 0.25 *$ & $14.95 \pm 4.10^{*}$ & $448.29 \pm 17.02$ & $17.37 \pm 1.93^{*}$ \\
\hline pH 10.8 buffer & $0.67 \pm 0.006^{* *}$ & $0.10 \pm 0.02 *$ & $1.57 \pm 0.36^{*}$ & $170.30 \pm 16.35^{*}$ & $5.85 \pm 4.32 *$ \\
\hline $10 \% \mathrm{GF}^{d)}$ in $\mathrm{pH} 6$ buffer & $1.03 \pm 0.07 * *$ & $2.32 \pm 0.29 * *$ & $22.57 \pm 2.78 *$ & $399.09 \pm 31.50$ & $248.36 \pm 25.87$ \\
\hline Soybean oil & $0.79 \pm 0.04 * *$ & $0.53 \pm 0.08 *$ & $9.91 \pm 1.53 *$ & $35.62 \pm 1.50^{*}$ & $17.79 \pm 4.85^{*}$ \\
\hline
\end{tabular}

a) Permeability coefficient=flux $\left(\mathrm{nmol} / \mathrm{cm}^{2} / \mathrm{h}\right) /$ solubility $\left.(\mathrm{nmol} / \mathrm{l}) . \quad b\right)$ Release rate $=$ the slope from released amount $\left(\mathrm{nmol} / \mathrm{cm}^{2} / \mathrm{h}\right)-$ time $\mathrm{profiles} / \mathrm{solubility}(\mathrm{nmol} / \mathrm{l})$ c) Calibrated skin deposition=drug amount in skin $(\mathrm{nmol} / \mathrm{mg}) /$ solubility $(\mathrm{nmol} / \mathrm{l}) . \quad d) \mathrm{GF}=$ glycerol formal. $*$ Significantly lower $(p<0.05)$ as compared to $\mathrm{pH} 6$ buffer group; ** significantly higher $(p<0.05)$ as compared to $\mathrm{pH} 6$ buffer group. The data represent the mean \pm S.D. $(n=4)$. 
Table 2. Permeability Coefficient and Skin Deposition of Resveratrol across the $\mathrm{SC}^{a}$-Stripped Skin

\begin{tabular}{lcccc}
\hline \hline Vehicle & $\begin{array}{c}\text { Permeability coefficient } \\
\left(\mathrm{cm} / \mathrm{h} \times 10^{-4}\right)^{b}\end{array}$ & Ratio $_{\mathrm{PC}}{ }^{c)}$ & $\begin{array}{c}\text { Skin deposition } \\
(\mu \mathrm{g} / \mathrm{g})^{d}{ }^{2}\end{array}$ & Ratio $\left._{\mathrm{SD}}{ }^{e}\right)$ \\
\hline pH 6 buffer & $77.80 \pm 11.59$ & 1.08 & $194.01 \pm 45.38$ & 0.64 \\
pH 8 buffer & $68.82 \pm 29.53$ & 0.86 & $233.06 \pm 102.14$ & 0.85 \\
pH 9.9 buffer & $112.36 \pm 20.64$ & 7.52 & $196.08 \pm 165.12$ & 11.29 \\
pH 10.8 buffer & $48.40 \pm 6.79$ & 30.83 & $132.28 \pm 48.59$ & 22.61 \\
\hline
\end{tabular}

a) $\mathrm{SC}=$ stratum corneum. b) Permeability coefficient $=$ flux $\left(\mathrm{nmol} / \mathrm{cm}^{2} / \mathrm{h}\right) /$ solubility $\left.(\mathrm{nmol} / \mathrm{l}) . \quad c\right)$ Ratio PC $_{\mathrm{PC}}$ permeability coefficient across SC-stripped skin/permeability

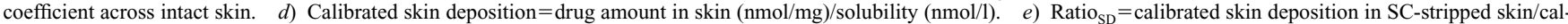
ibrated skin deposition in intact skin. The data represent the mean \pm S.D. $(n=4)$.

Table 3. Permeability Coefficient and Skin Deposition of Resveratrol across the Skin Treated by Different Methods

\begin{tabular}{|c|c|c|c|c|}
\hline Treatment & $\begin{array}{l}\text { Permeability coefficient } \\
\qquad\left(\mathrm{cm} / \mathrm{h} \times 10^{-4}\right)^{a)}\end{array}$ & Ratio $\left._{\mathrm{PC}}{ }^{b}\right)$ & $\begin{array}{l}\text { Skin deposition } \\
\qquad(\mu \mathrm{g} / \mathrm{g})^{c)}\end{array}$ & Ratio $_{S D}{ }^{d)}$ \\
\hline Delipid & $78.49 \pm 7.21$ & 1.09 & $193.85 \pm 35.95$ & 0.66 \\
\hline $25 \%$ Ethanol & $63.89 \pm 7.05$ & 0.89 & $295.37 \pm 118.62$ & 0.97 \\
\hline$\alpha$-Terpineol in $25 \%$ ethanol & $81.73 \pm 10.95^{* *}$ & $1.28^{e)}$ & $228.72 \pm 82.21$ & $0.77^{e)}$ \\
\hline Oleic acid in $25 \%$ ethanol & $65.44 \pm 6.69$ & $1.02^{e)}$ & $163.65 \pm 57.98^{*}$ & $0.55^{e)}$ \\
\hline
\end{tabular}

a) Permeability coefficient $=$ flux $\left(\mathrm{nmol} / \mathrm{cm}^{2} / \mathrm{h}\right) /$ solubility $(\mathrm{nmol} / \mathrm{l})$. b) Ratio $\mathrm{PC}_{\mathrm{C}}=$ permeability coefficient across the treated skin/permeability coefficient across intact skin

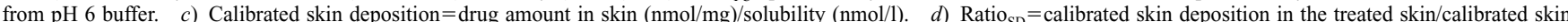
deposition in intact skin from $\mathrm{pH} 6$ buffer. e) The ratio is calibrated by the data of $25 \%$ ethanol-treated group. * Significantly lower $(p<0.05)$ as compared to $25 \%$ ethanol group; ** significantly higher $(p<0.05)$ as compared to $25 \%$ ethanol group. The data represent the mean \pm S.D. $(n=4)$.

ity in glycerol formal. Soybean oil, a lipophilic vehicle, was also used for resveratrol permeation. Resveratrol showed higher solubility in soybean oil compared to the aqueous buffers $(p<0.05)$. Resveratrol should act as a non-ionic form in the oil. The skin permeation and deposition of the drug with soybean oil were relatively low compared to those from $\mathrm{pH} 6$ buffer in which resveratrol was neutralized.

In Vitro Skin Permeation of Resveratrol across Skins with Different Treatments In order to elucidate the mechanisms involved in the skin permeation of resveratrol, in vitro permeation experiments were performed using various skin membranes. Aqueous buffers were used as the vehicles because of their good permeability for resveratrol. Table 2 summarizes the $K_{\mathrm{p}}$ and skin deposition via $\mathrm{SC}$-stripped skin from buffers with various $\mathrm{pH}$ values. The enhancements of permeation and deposition generally increased as the $\mathrm{pH}$ increased after stripping the SC. There was no significant difference ( $p>0.05)$ between the $K_{\mathrm{p}}$ values with and without the SC at $\mathrm{pH} 6$ and 8. Removal of the SC even reduced the skin deposition of resveratrol $(p<0.05)$ in the non-ionized form $(\mathrm{pH} 6)$.

The skin permeation of resveratrol with $\mathrm{pH} 6$ buffer across delipidized skin is shown in Table 3. Similar $K_{\mathrm{p}}$ values of permeation across delipidized and SC-stripped skin were observed. $\alpha$-Terpineol and oleic acid are commonly used as permeation enhancers. Pretreatments with both of these enhancers on the skin did not further increase the permeation of resveratrol in non-ionized form as shown in Table 3 ( $\mathrm{pH} \mathrm{6)}$. Drug permeation with $\alpha$-terpineol was slightly but significantly higher $(p<0.05)$ than that with oleic acid. Delipidized and enhancer-pretreated skin exhibited lower resveratrol accumulation within the reservoir compared to intact skin.

In Vitro Skin Permeation of Resveratrol from Hydrogels The patch and semisolid forms are more applicable than a solution as a transdermal delivery system for clinical administration. Hence hydrogels with various polymers were used as vehicles to examine resveratrol permeation as shown in Fig. 3. Among the hydrogels with a polymer concentration

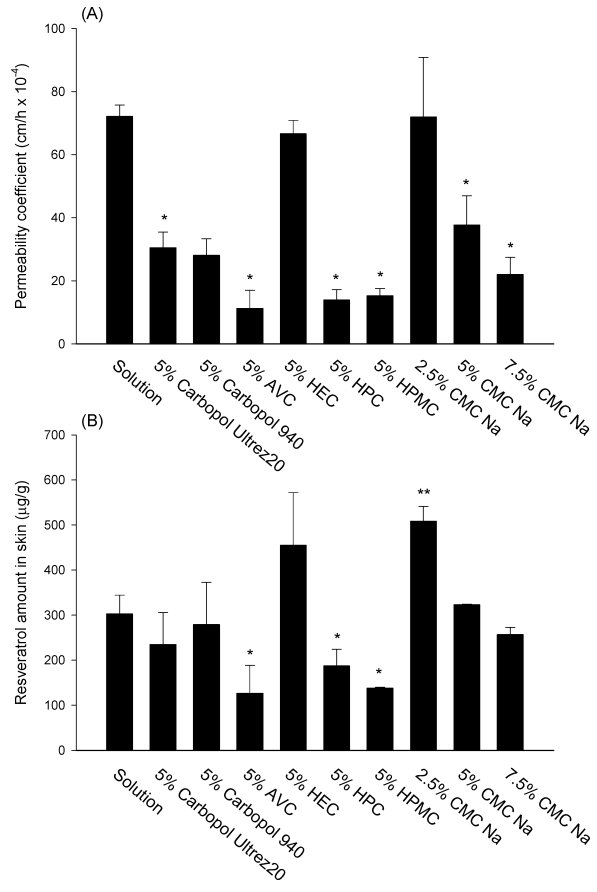

Fig. 3. Permeability Coefficient (A) and Skin Deposition in the Skin Reservoir (B) of in Vitro Topical Resveratrol Application Permeating across Nude Mouse Skin from Different Hydrogel Matrices

All data represent the means of four experiments \pm S.D. * Significantly lower $(p<0.05)$ as compared to solution; ** significantly higher $(p<0.05)$ as compared to solution.

of $5 \%$, the formulation with HEC exhibited the greatest $K_{\mathrm{p}}$ value (Fig. 3A), followed by $\mathrm{CMCNa}$ and the Carbopol polymers. The hydrogels with AVC, HPC, or HPMC showed the lowest drug penetration values with no significant differences among them $(p>0.05)$. CMCNa was chosen as the model vehicle to further investigate the influence of polymer contents on drug permeation. As demonstrated in Fig. 3A, resveratrol permeation decreased with an increase in the content of 


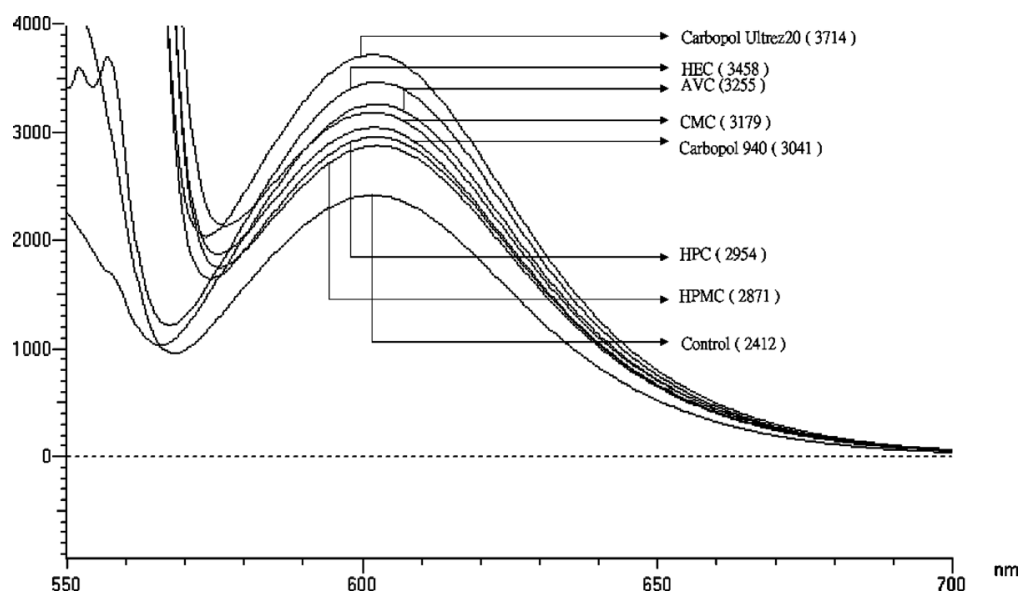

Fig. 4. Fluorescence Emission Spectra of Nile Red $\left(2.5 \times 10^{-5} \%\right.$, w/w) in Different Hydrogels with $5 \%$ Polymer

The datum behind the polymer type is the fluorescence intensity at a wavelength of $602 \mathrm{~nm}\left(\lambda_{\max }\right)$.

CMCNa in the gels. The trend of drug deposition in the skin from hydrogels was similar to that of $K_{\mathrm{p}}$ (Fig. 3B).

The physicochemical properties of hydrogels, including polarity and viscosity, were examined. Nile red is a dye whose absorption bands vary in shape, position, and intensity with the nature of the environment. The emission spectra of Nile red in the hydrogels are shown in Fig. 4. The fluorescence intensity was monitored at a wavelength of $602 \mathrm{~nm}$, the emission maximum of Nile red. The fluorescence is quenched in more-hydrophilic environments. ${ }^{15)}$ The $\mathrm{pH} 6$ buffer without polymers (control) showed the least intense fluorescence. The addition of polymers raised the intensity to different levels, with Carbopol Ultrez20 showing the highest value.

The viscosities of the hydrogels are summarized in Table 4. The HEC hydrogel, which showed the highest resveratrol permeation among all hydrogels tested, possessed the lowest viscosity. The two Carbopol hydrogels exhibited large discrepancies between their viscosities (15.54 vs. $36.33 \mathrm{~Pa} \cdot \mathrm{s}$ for Carbopol Ultrez20 and 940, respectively). The viscosity increased following an increase in the $\mathrm{CMCNa}$ percentage in the gel matrix.

In Vitro Skin Permeation of Piceatannol Piceatannol is an analogue of resveratrol which has strong anti-inflammatory and anticancer activities. ${ }^{16,17)}$ The solubility and permeation/deposition profiles of piceatannol were established for comparison with those of resveratrol as shown in Table 5. Piceatannol showed a higher aqueous solubility $(p<0.05)$ in pH 6 buffer than resveratrol $(0.94 v s .0 .22 \mathrm{nmol} / 1)$. The solubility of piceatannol in soybean oil was extremely low. The $K_{\mathrm{p}}$ value of piceatannol at $\mathrm{pH} 6$ was 11.6 -fold lower than that of resveratrol across intact skin. Stripping of the SC enhanced piceatannol permeation by 2.7 -fold. No piceatannol permeated into the receptor when using soybean oil as the drug vehicle, although high skin deposition was obtained after calibration by solubility.

In Vivo Skin Permeation of Resveratrol As shown in Table 6, the in vivo uptake of resveratrol followed an increasing trend of $\mathrm{pH} 6$ buffer $>\mathrm{CMCNa}$ hydrogel $>$ soybean oil. This trend is similar to the results of in vitro permeation. Bioengineering methods such as TEWL, colorimetry, and $\mathrm{pH}$ value for evaluating the preliminary safety of resveratrol and
Table 4. Viscosity $(\mathrm{Pa} \cdot \mathrm{s})$ of the Hydrogels

\begin{tabular}{lc}
\hline \hline \multicolumn{1}{c}{ Polymer } & Viscosity $(\mathrm{Pa} \cdot \mathrm{s})$ \\
\hline $5 \%$ Carbopol Ultrez20 & $15.54 \pm 0.47$ \\
$5 \%$ Carbopol 940 & $36.33 \pm 0.61$ \\
$5 \%$ AVC & $45.24 \pm 0.57$ \\
$5 \%$ HEC & $5.08 \pm 0.34$ \\
$5 \%$ HPC & $36.31 \pm 2.97$ \\
$5 \%$ HPMC & $34.57 \pm 0.65$ \\
2.5\% CMC Na & $0.16 \pm 0.02$ \\
$5 \%$ CMC Na & $20.93 \pm 0.39$ \\
$7.5 \% \mathrm{CMC} \mathrm{Na}$ & $23.55 \pm 0.96$ \\
\hline
\end{tabular}

The data represent the mean \pm S.D. $(n=3)$.

its formulations were conducted in vivo. The $\Delta$ values (the value of the treated site minus the value of an adjacent untreated site) of TEWL, $\mathrm{a}^{*}$, and $\mathrm{pH}$ were determined after 24$\mathrm{h}$ applications of the topical formulations. Table 6 shows that the $\mathrm{pH} 6$ buffer demonstrated a significant increase $(p<0.05)$ in the extent of water loss from the SC $\left(16.55 \mathrm{~g} / \mathrm{m}^{2} / \mathrm{h}\right)$ relative to the control. Treatments with oil and hydrogel produced no enhancement of $\triangle$ TEWL $(p>0.05)$. The results of $\Delta \mathrm{a}^{*}$ showed that the three systems produced negligible erythema. A similar result was observed for the determination of the skin surface $\mathrm{pH}$.

\section{DISCUSSION}

The drug solution at saturation was used for the skin permeation experiment to ensure that there was uniform thermodynamic activity and thus common activity for the drug in each formulation. ${ }^{18)}$ The $K_{\mathrm{p}}$ value is independent of the donor concentration, unlike the flux, so this parameter is important for comparing permeation levels among various formulations. A higher observed $K_{\mathrm{p}}$ for a drug indicates its superiority for topical/transdermal delivery. The results in Table 1 suggest that the permeation of resveratrol was highly dependent upon the $\mathrm{pH}$ of the aqueous vehicle. Resveratrol in completely or partially neutral condition showed higher permeability compared to the ionized condition. A similar result was observed for profiles of drug skin deposition.

The skin permeation of a drug is determined by its physic- 
Table 5. Solubility, Permeation Data, and Skin Deposition of Piceatannol from $\mathrm{pH} 6$ Buffer and Soybean Oil

\begin{tabular}{|c|c|c|c|c|c|}
\hline Vehicle & Skin type & $\begin{array}{l}\text { Solubility } \\
(\mathrm{nmol} / \mathrm{l})\end{array}$ & $\begin{array}{c}\text { Flux } \\
\left(\mathrm{nmol} / \mathrm{cm}^{2} / \mathrm{h}\right)\end{array}$ & $\begin{array}{l}\text { Permeability coefficient or } \\
\left.\text { release rate }\left(\mu \mathrm{m} / \mathrm{h} \times 10^{-4}\right)^{a}\right)\end{array}$ & $\begin{array}{l}\text { Skin deposition } \\
\qquad(\mu \mathrm{g} / \mathrm{g})^{b)}\end{array}$ \\
\hline pH 6 buffer & Intact skin & $0.94 \pm 0.18$ & $0.58 \pm 0.21$ & $6.20 \pm 2.25$ & $9.42 \pm 6.13$ \\
\hline pH 6 buffer & $\mathrm{SC}^{c)}$-stripped skin & $0.94 \pm 0.18$ & $1.59 \pm 0.25^{* *}$ & $16.91 \pm 2.62 * *$ & $25.38 \pm 7.70 * *$ \\
\hline Soybean oil & Intact skin & $0.005 \pm 0.001 *$ & 0 & 0 & $133.49 \pm 29.36 * *$ \\
\hline
\end{tabular}

a) Permeability coefficient $=$ flux $\left(\mathrm{nmol} / \mathrm{cm}^{2} / \mathrm{h}\right) /$ solubility $(\mathrm{nmol} / 1)$; release rate $=$ the slope from released amount $\left(\mathrm{nmol} / \mathrm{cm}^{2} / \mathrm{h}\right)-$ time $\left.\mathrm{profiles} / \mathrm{solubility}(\mathrm{nmol} / \mathrm{l}) . \quad b\right)$ Calibrated skin deposition=drug amount in skin $(\mathrm{nmol} / \mathrm{mg}) /$ solubility $(\mathrm{nmol} / \mathrm{l}) . \quad c) \mathrm{SC}=$ stratum corneum. $*$ Significantly lower $(p<0.05)$ as compared to $\mathrm{pH} 6$ buffer group; $* *$ significantly higher $(p<0.05)$ as compared to $\mathrm{pH} 6$ buffer group. The data represent the mean \pm S.D. $(n=4)$.

Table 6. In Vivo Skin Deposition and Physiological Parameters of Resveratrol from Various Vehicles

\begin{tabular}{lrrr}
\hline \hline \multicolumn{1}{c}{ Parameter } & pH 6 buffer & Soybean oil & \multicolumn{1}{c}{$5 \%$ CMC Na } \\
\hline${\text { Skin deposition }(\mu \mathrm{g} / \mathrm{g})^{a)}}^{2}$ & $40.97 \pm 26.26$ & $3.96 \pm 3.37$ & $16.43 \pm 4.53$ \\
$\Delta \mathrm{TEWL}^{b)}\left(\mathrm{g} / \mathrm{m}^{2} / \mathrm{h}\right)$ & $16.55 \pm 2.23$ & $0.75 \pm 1.62$ & $-2.73 \pm 3.47$ \\
$\Delta \mathrm{a}^{*}$ & $-0.27 \pm 1.79$ & $-2.41 \pm 2.45$ & $0.35 \pm 1.36$ \\
$\Delta \mathrm{pH}$ & $-0.39 \pm 0.38$ & $-0.02 \pm 0.56$ & $-0.14 \pm 0.26$ \\
\hline
\end{tabular}

a) Calibrated skin deposition= drug amount in skin $(\mathrm{nmol} / \mathrm{mg}) /$ solubility $(\mathrm{nmol} / \mathrm{l})$ b) $\mathrm{TEWL}=$ transepidermal water loss. The data represent the mean \pm S.D. $(n=6)$

ochemical properties, in particular the molecular weight and lipophilicity, which play major roles in the process. ${ }^{19)}$ More-lipophilic components may penetrate the SC more readily. There is also good evidence that the $\mathrm{SC}$ is much more permeable to neutral molecules than to ions of weak acids or bases. ${ }^{20,21)}$ The non-ionic form of resveratrol shows high lipophilicity according to the partition coefficient $\left(\log \mathrm{P}_{\text {octanol } / \text { water }}=3.32\right)$. The partition coefficient for compounds between $\mathrm{SC}$ and water $\left(\log \mathrm{P}_{\mathrm{SC} / \text { water }}\right)$ can be estimated as $^{22)}: \log \mathrm{P}_{\mathrm{SC} / \text { water }}=-0.024+0.59 \log \mathrm{P}_{\text {octanol/water. }}$ This formula shows that the partitioning of the drug into the $\mathrm{SC}$ from an aqueous solution is directly related to its lipophilicity. Hence resveratrol is feasible as a candidate for skin absorption in the neutral condition.

With respect to drug permeation across the skin from the vehicle, a drug should first diffuse out of the vehicle onto the skin surface. The release rate of resveratrol was significantly higher than the $K_{\mathrm{p}}$ values. This indicates that the skin exerts a significant barrier function against the transport of resveratrol. The drug in $\mathrm{pH} 10.8$ buffer showed a slower release rate compared to that in other buffer systems. Since the thermodynamic activity was identical for all systems, the reduced release rate at $\mathrm{pH} 10.8$ might not have been due to the diffusivity of the drug but to other possible factors such as resveratrol being adsorbed by cellulose materials. ${ }^{13)}$ However, further investigation is needed to elucidate this inference.

The incorporation of glycerol formal in the $\mathrm{pH} 6$ buffer significantly reduced the permeation of resveratrol. The addition of a cosolvent may reduce the polarity of the aqueous vehicles. The increasing solubility with cosolvent incorporation in the vehicle hinders the partitioning of the drug into the SC. ${ }^{2324)}$ Soybean oil showed low resveratrol permeation and skin deposition. As the oil is relatively lipophilic, the partitioning of resveratrol would be expected to favor the vehicle. Another explanation is the slow rate of drug release from soybean oil. This may be due to the higher viscosity of the oil relative to the aqueous solution, making it difficult for the drug to diffuse within the oil system.

The SC is principally lipophilic in nature and far more re- sistant to polar than non-polar compounds. This speculation is consistent with the permeation profiles of resveratrol with the removal of the SC which largely increased drug permeation and deposition in the ionic form ( $\mathrm{pH} \mathrm{9.9,10.8).} \mathrm{This} \mathrm{in-}$ dicates that the $\mathrm{SC}$ is a major contributor to the barrier function against skin permeation of anionic resveratrol. SC stripping did not change resveratrol's permeation at lower $\mathrm{pH}$ values. Moreover, the $K_{\mathrm{p}}$ value of resveratrol across SC-stripped skin was relatively lower than the release rate. These results suggest that drug permeation might be hindered not by the $\mathrm{SC}$, but by the layers of the epidermis/dermis beneath the SC. As the drug approaches the extremes of high lipophilicity, the aqueous tissue resistance such as by viable skin becomes the predominant source of resistance. ${ }^{25,26)}$ Ablation of the $\mathrm{SC}$ even reduced the skin deposition at $\mathrm{pH}$ 6. This may have been due to a lack of the SC drug reservoir within the skin, resulting in insufficient space for the accumulation of the drug. ${ }^{27)}$ This indicates the importance of the SC as a skin reservoir for non-ionic resveratrol.

Permeation through corneocytes (i.e., a transcellular pathway) and permeation through the lipid bilayers surrounding the corneocytes (i.e., an intercellular pathway) contribute to the routes for drug permeation across the SC. The relative importances of the two pathways for drug delivery can be evaluated using delipidized skin as the barrier. For a drug which mainly permeates through the lipid bilayer region, similar $K_{\mathrm{p}}$ values should be observed for those transported across SC-stripped and dilipidized skin. The present study showed such results, suggesting that the predominant route is in the intercellular region. The $K_{\mathrm{p}}$ value of anionic resveratrol across delipidized skin at $\mathrm{pH} 10.8$ was also determined to be $33.80 \times 10^{-4} \mathrm{~cm} / \mathrm{h}$, which was lower than the value across SC-stripped skin $\left(48.40 \times 10^{-4} \mathrm{~cm} / \mathrm{h}\right)$. This indicates that the intracellular route partly contributes to the permeation pathway of the anionic form. This result somewhat differs from that of the neutral form.

Both $\alpha$-terpineol and oleic acid are permeation enhancers for transdermal drug delivery. Terpenes have been shown to act at the lipid polar heads of ceramides, ${ }^{28)}$ while fatty acids act at the lipidic tail portion of intercellular lipid bilayers. ${ }^{29}$ ) A $25 \%$ ethanol in water mixture was used as the pretreatment medium for both enhancers for solubility considerations. Neither enhancers could elaborate their permeation-enhancing activity on resveratrol transport. Oleic acid also has an effect on the drug's partitioning into the SC. ${ }^{30)}$ This suggests that resveratrol might be easily partitioned into the SC with no need to increase the partitioning by enhancers. The results confirm the success of topical/transdermal delivery of nonionic resveratrol because of its excellent permeability in the absence of enhancing methods. 
In case of application of solutions on the skin, it is difficult to expect their effects to be prolonged for a significant period of time because they are easily removed by wetting, movement, and contact. ${ }^{31)}$ Hence bioadhesive hydrogels or patches that have good accessibility can be developed for localized administration. Based on the data of resveratrol permeation from solutions, an aqueous system was superior to an oily system because of the higher permeability of resveratrol. Hydrogels may be feasible as vehicles for resveratrol due to the high percentage of water in the systems. Moreover, as patches may be associated with local skin irritation, gel formulations have been developed to improve compliance with transdermal delivery systems. ${ }^{32)}$ To yield promise for further applications, polymers were added to the aqueous vehicles to form hydrogel matrices. All hydrogels with 5\% polymer showed lower $K_{\mathrm{p}}$ values compared to the $\mathrm{pH} 6$ buffer except for HEC, which exhibited higher drug permeation among the hydrogels. The drug contained in such matrices should not be readily absorbed into the skin as it is not in a fluid state. The $K_{\mathrm{p}}$ value of resveratrol from the HEC hydrogel was comparable to that from an aqueous solution, indicating that the cross-linkage structure formed by 5\% HEC did not interact with the drug. HEC might not be applicable because of its low viscosity as determined in this study.

CMCNa showed moderate viscosity and good bioadhesion to the skin. ${ }^{33)}$ As shown in the permeation results from CMCNa hydrogels with different polymer concentrations, the increased polymer content may result in higher entanglement density and thus decreased permeation rates. It is thought that such polymers in gels function as a diffusion barrier to influence drug permeation. This result suggests that the diffusion of the drug through hydrogel vehicles is the predominant mechanism controlling the permeation process.

Resveratrol release across cellulose membranes from hydrogels was investigated to elucidate whether the diffusion within hydrogels was important (data not shown). A significant correlation was detected (Pearson's correlation $r=$ $0.9000, p<0.001)$ between the data of in vitro $K_{\mathrm{p}}$ values and the release rate from hydrogels. This suggests that release from the gel matrix may be the rate-determining step controlling drug permeation into the receptor compartment. A correlation existed between the resveratrol skin content and the $K_{\mathrm{p}}$ value from hydrogels $(r=0.9760, p<0.001)$. A significant relationship between skin deposition and permeation was also observed in aqueous buffer systems $(r=0.9874$, $p<0.001)$. This indicated that the drug localized in the skin may retained the drug and permitted its diffusion and final permeation.

The polarity and cross-linking structure are characteristics of hydrogels which may influence drug permeation. A previous study presented a trend of increasing hydrophilicity in the order of $\mathrm{CMCNa}>\mathrm{HEC}>\mathrm{HPC}>\mathrm{HPMC}$ for cellulose polymers. ${ }^{34)}$ Those results greatly differed from ours. We examined the polarity of these polymers in a hydrogel form by the Nile red method. Nile red is very soluble in organic solvents such as acetone and strongly fluorescent in a lipophilic environment. Nile red emission shifts to longer wavelengths indicate an increase in environmental polarity. ${ }^{15)}$ A reduction in the fluorescent intensity at $c a$. $600 \mathrm{~nm}$ also indicates a polarity increment because the fluorescence is quenched in a more hydrophilic environment. The water-polymer interac- tion and addition of components such as buffer species may alter the intermolecular forces, free volume, and swelling properties of the hydrogel polarity. Moreover, the molecular weights and chain orders of one polymer produced by different manufacturers showed a large divergence. Since resveratrol is lipophilic at $\mathrm{pH} 6$, an increase in the hydrogel lipophilicity might lead to difficulty of resveratrol diffusion because of the drug-hydrogel affinity. Unfortunately, a precise linear relationship was not established between hydrogel polarity and the permeability coefficient $(r=0.5904, p=$ $0.123)$.

Viscosity is the most widely utilized reference for characterizing a hydrogel's structure, although it is not sufficiently comprehensive for the full determination of a hydrogel's strength. As a general rule, an increase in the viscosity of the vehicle should produce a more-rigid structure and decrease the rate of drug permeation. The correlation between viscosity and $K_{\mathrm{p}}$ was significant for the hydrogels $(r=0.8902$, $p=0.006$ ), suggesting that viscosity played an important role in controlling the drug release and subsequent permeation.

Piceatannol was found to have antioxidant activity similar to that of resveratrol, but higher radical-scavenging activity. ${ }^{35)}$ Piceatannol has an additional hydroxyl group in the $3^{\prime}$-position of the structure compared to resveratrol. The extra moiety contributes to the higher aqueous solubility of piceatannol than resveratrol. The skin permeation of piceatannol was also less because of its more-hydrophilic properties compared to resveratrol. The result of permeation across the SC-stripped skin suggests that a barrier function of the SC exists for piceatannol but not for resveratrol. The unique permeation profile of piceatannol surpassed that of resveratrol in skin deposition with the oil. Piceatannol may tend to escape from the oil to the skin due to the low affinity of lipophilic oil for piceatannol.

A good in vitro-in vivo correlation was observed for resveratrol permeation from various systems. The in vivo skin uptake showed a less-close relationship with the in vitro skin deposition $(r=0.7188, p=0.489)$ than that with the in vitro $K_{\mathrm{p}}(r=0.9923, p=0.079)$. This possibly suggests that the amount of resveratrol permeating into the receptor in the in vitro status can predict the in vivo drug accumulation within the skin reservoir. However, the in vivo skin deposition was much less than the in vitro result. This may be due to the significant diffusion and distribution of the drug from skin to the systemic circulation or other tissues after in vivo topical administration, thus reducing the skin accumulation in the in vivo status.

TEWL is used to assess the degree of disruption of the $\mathrm{SC}$; and a good correlation between the chemical damage to the skin barrier and an increase in TEWL has been demonstrated. On the other hand, the $\mathrm{a}^{*}$-coordinate of colorimetry (which indicates erythema) has been demonstrated to correlate well with inflammatory interactions of the skin, especially in viable skin. ${ }^{36)}$ None of the vehicles showed any or only negligible increases in the TEWL, a* value, and $\mathrm{pH}$ of skin except for the increased TEWL caused by the $\mathrm{pH} 6$ buffer. This may suggest a good tolerance of topical resveratrol on skin in this preliminary safety test. This may have been due to the high amount of the aqueous phase in the buffer hydrating the SC, producing evaporation of excess water after removing the vehicle in an occlusive condition. 
The $\mathrm{pH} 6$ buffer without resveratrol was also used for the in vivo safety study. A $\Delta$ TEWL value $\left(15.50 \pm 1.39 \mathrm{~g} / \mathrm{m}^{2} / \mathrm{h}\right) \mathrm{sim}-$ ilar to that produced by the vehicle with the drug was detected, indicating that the buffer, but not resveratrol, induced this high TEWL value. No damage to the skin occurred as a result of hydrogel treatment. The polymer in the hydrogel may hold the water within the vehicle, preventing excess hydration of the skin by the water. It can be concluded that the hydrogel may be an ideal system for resveratrol delivery because it provides a high level of drug permeation and skin safety. Further safety test is needed and in progress, e.g. histological assay and ultrastructural examination by electric microscopy.

\section{CONCLUSIONS}

Despite good partitioning into the SC, the permeability of highly lipophilic molecules is always low. This is probably due to the accumulation of lipophilic drugs in the SC. This phenomenon was not observed with non-ionic resveratrol, which showed higher permeability via the skin. These results support the superiority of resveratrol for both topical and transdermal delivery. Resveratrol transport via the skin was closely related to the vehicle in which it was formulated, with aqueous buffers with lower $\mathrm{pH}$ values exhibiting good permeability/deposition. The viable epidermis/dermis but not the SC layer acted as a diffusion barrier for resveratrol permeation. Piceatannol, a more-hydrophilic analogue of resveratrol, showed lower permeation compared to resveratrol. Resveratrol delivery by skin route may avoid the degradation because the low metabolism in the skin, resulting in the possible prolongation of half-life and sufficient concentration in the systemic circulation. Moreover, resveratrol retained within the skin after topical application can be an efficient way to be a therapy or prevention of UV exposure and skin carcinogenesis. Further study is, of course, needed to confirm these efficiencies. This study indicates the promise of further in vivo and clinical applications of resveratrol delivery via the skin.

\section{REFERENCES}

1) Kaldas M. I., Walle U. K., Walle T., J. Pharm. Pharmacol., 55, 307312 (2003).

2) Jang M., Pezzuto J. M., Cancer Lett., 134, 81-89 (1998).

3) Soleas G. J., Grass L., Josephy P. D., Goldberg D. M., Diamandis E. P., Clin. Biochem., 35, 119-124 (2002).

4) Aziz M. H., Reagan-Shaw S., Wu J., Longley B. J., Ahmad N., FASEB J., 19, 1193-1195 (2005).

5) Afaq F., Adhami V. M., Ahmad N., Toxicol. Appl. Pharmacol., 186, $28-37$ (2003).
6) Aziz M. H., Afaq F., Ahmad N., Photochem. Photobiol., 81, 25-31 (2005).

7) Chan M. M. Y., Biochem. Pharmacol., 63, 99-104 (2002).

8) Docherty J. J., Smith J. S., Fu M. M., Stoner T., Booth T., Antiviral Res., 61, 19-26 (2004).

9) Bowers J. L., Tyulmenkov V. V., Jernigan S. C., Klinge C. M., Endocrinology, 141, 3657-3667 (2000).

10) Sale S., Verschoyle R. D., Boocock D., Jones D. J. L., Wilsher N., Ruparelia K. C., Potter G. A., Farmer P. B., Steward W. P., Gescher A. J., Br. J. Cancer, 90, 736-744 (2004).

11) Baur J. A., Sinclair D. A., Nature Drug Discover, 5, 493-506 (2006).

12) Shin S. C., Kim H. J., Oh I. J., Cho C. W., Yang K. H., Eur. J. Pharm. Biopharm., 60, 67-71 (2005).

13) Takagai Y., Kubota T., Kobayashi H., Tashiro T., Takahashi A., Igarashi S., Anal. Sci., 21, 183-186 (2005).

14) Hung C. F., Chen J. K., Liao M. H., Lo H. M., Fang J. Y., J. Nanosci. Nanotechnol., 6, 2950-2958 (2006).

15) Jores K., Haberland A., Wartewig S., Mäder K., Mehnert W., Pharm. Res., 22, 1887-1897 (2005).

16) Wieder T., Prokop A., Bagci B., Essmann F., Bernicke D., SchulzeOsthoff K., Dorken B., Schmalz H. G., Daniel P. T., Henze G., Leukemia, 15, 1735-1742 (2001).

17) Djoko B., Chiou R. Y. Y., Shee J. J., Liu Y. W., J. Agric. Food Chem., 55, 2376-2383 (2007).

18) Imoto H., Zhou Z., Stinchcomb A. L., Flynn G. L., Biol. Pharm. Bull., 19, 263-267 (1996).

19) Marti-Mestres G., Mestres J. P., Bres J., Martin S., Ramos J., Vian L., Int. J. Pharm., 331, 139-144 (2007).

20) Swarbrick J., Lee B., Brom J., Gensmantel N. P., J. Pharm. Sci., 73, 1352-1355 (1984).

21) Fang J. Y., Tsai T. H., Lin Y. Y., Wong W. W., Wang M. N., Huang J. F., Biol. Pharm. Bull., 30, 343-349 (2007).

22) Roberts M. S., Pugh W. J., Hadgraft J., Int. J. Pharm., 132, 23-42 (1996).

23) Fang J. Y., Hwang T. L., Leu Y. L., Int. J. Pharm., 250, 313-325 (2003).

24) Nicolazzo J. A., Morgan T. M., Reed B. L., Finnin B. C., J. Controlled Release, 103, 577-585 (2005)

25) Stinchcomb A., Paliwal A., Dua R., Imoto H., Woodard R. W., Flynn G. L., Pharm. Res., 13, 1519-1523 (1996).

26) Sung K. C., Fang J. Y., Wang J. J., Hu O. Y. P., Eur. J. Pharm. Sci., 18, $63-70$ (2003).

27) Lee W. R., Shen S. C., Wang K. H., Hu C. H., Fang J. Y., J. Invest. Dermatol., 121, 1118-1125 (2003).

28) Panchagnula R., Desu H., Jain A., Kriandarilli S., J. Pharm. Sci., 93, $2177-2183$ (2004).

29) Jain A. K., Panchagnula R., Meth. Find. Exp. Clin. Pharmacol., 25, 413-421 (2003)

30) Moser K., Kriwet K., Naik A., Kalia Y. N., Guy R. H., Eur. J. Pharm. Biopharm., 52, 103-112 (2001).

31) Shin S. C., Kim H. J., Oh I. J., Cho C. W., Yang K. H., Eur. J. Pharm. Biopharm., 60, 67-71 (2005).

32) Samsioe G., Climacteric, 7, 347-356 (2004).

33) Jones D. S., Woolfson A. D., Brown A. F., Int. J. Pharm., 151, 223233 (1997).

34) Narasimhan B., Peppas N. A., J. Pharm. Sci., 86, 297-304 (1997).

35) Fauconneau B., Waffo-Teguo P., Huguet F., Barrier L., Decendit A., Merillon J. M., Life Sci., 61, 2103-2110 (1997).

36) Zhao K., Singh J., J. Controlled Release, 62, 359-366 (1999). 